\title{
Perfil epidemiológico de pacientes com metástase na coluna vertebral em Salvador, Bahia, Brasil
}

\section{Epidemiological Profile of Patients with Spinal Metastases in Salvador, Bahia, Brazil}

\author{
Reinaldo Rodrigues Pamplona ${ }^{1}$ Bruno Muniz Pinto² \\ ${ }^{1}$ Médico Assistente em Neurocirurgia do Hospital Geral Roberto \\ Santos, Salvador, BA, Brasil \\ ${ }^{2}$ Acadêmico de Medicina, Faculdade de Medicina, Universidade \\ Federal da Bahia, Salvador, BA, Brasil \\ ${ }^{3}$ Médico Residente em Neurocirurgia, Santa Casa de Misericórdia de \\ Ribeirão Preto, SP, Brasil \\ ${ }^{4}$ Médico Plantonista, Hospital Geral Roberto Santos, Salvador, \\ BA, Brasil
}

George Santos dos Passos ${ }^{3}$ Leo Gordiano Matias ${ }^{4}$

Address for correspondence Reinaldo Rodrigues Pamplona, MD, Rua Manoel Andrade, 85, Pituba, Condomínio Pituba Ville, Apt 508, Salvador,

Arq Bras Neurocir 2016;35:8-12.

\section{Resumo \\ Palavras-Chave \\ - coluna vertebral \\ - neoplasias \\ - metástase neoplásica \\ - neoplasias da coluna vertebral \\ - biópsia por agulha}

Objetivo Analisar o perfil epidemiológico dos pacientes com suspeita de doença metastática na coluna vertebral em hospital de referência.

Métodos Estudo observacional, descritivo e retrospectivo, realizado em hospital de referência neurocirúrgica do estado da Bahia, a partir de dados coletados em prontuários de pacientes submetidos à biópsia percutânea da coluna vertebral por suspeita de patologia metastática no período de março de 2013 a fevereiro de 2014. Resultados Foram incluídos 46 pacientes, sendo 25 (54,3\%) do sexo masculino, com idade média de 54,3 anos (desvio padrão: 15 ). Mais da metade dos pacientes (56,2\%) possuíam diagnóstico de neoplasia anterior à biópsia vertebral, sendo os tipos mais comuns o adenocarcinoma de mama e próstata, seguidos pela neoplasia pulmonar. A coluna lombar foi a mais frequentemente acometida $(n=31 ; 67,4 \%)$, seguida pela torácica $(n=14 ; 30,4 \%)$ e sacral $(n=11 ; 23,9 \%)$. Nenhum paciente apresentou acometimento cervical em nossa amostra. $\mathrm{O}$ número de vértebras acometidas variou de 1 a 4 , sendo que 8 pacientes $(17,4 \%$ ) apresentavam lesões múltiplas ( $\geq 3$ lesões). 0 mieloma múltiplo foi a neoplasia mais comumente encontrada na análise do material de biópsia vertebral, correspondendo a 12 casos $(26,1 \%)$, seguido pela metástase de carcinoma de mama, 9 (19,6\%); de próstata, 5 (10,9\%); e pulmonar, 5 (10,9\%). Sete pacientes $(15,2 \%)$ não apresentaram alterações neoplásicas.

Conclusões Foram encontrados dados epidemiológicos compatíveis com a literatura, exceto pelo tipo histológico das metástases vertebrais, sendo mieloma múltiplo o de maior prevalência em nosso estudo. Este estudo torna-se relevante por ser o primeiro a avaliar metástases vertebrais no Norte-Nordeste brasileiro. received

May 17, 2015

accepted

August 28, 2015

published online

February 16, 2016
DOI http://dx.doi.org/

$10.1055 / \mathrm{s}-0035-1571209$.

ISSN 0103-5355.
Copyright $(2016$ by Thieme Publicações License terms

Ltda, Rio de Janeiro, Brazil $\circledast(1) \Theta$ 
Abstract

Keywords
- spine
- neoplasms
- neoplasm
metastasis
- spinal neoplasms
- biopsy
- needle

Objective To analyze the epidemiological profile of patients with suspected spine metastases in a referral hospital.

Methods An observational, descriptive and retrospective study, conducted in neurosurgical referral hospital in the state of Bahia with data collected from medical records of patients undergoing percutaneous spinal biopsy for suspected metastatic disease from March 2013 to February 2014.

Results 46 were included, which 25 (54.3\%) were male. The mean age was 54.3 years (SD: 15$)$. More than half of patients (56.2\%) had a previous diagnosis of cancer; the most common types were breast and prostate adenocarcinoma, followed by lung cancer. The lumbar spine was the most frequently affected $(n=31,67.4 \%)$, followed by the thoracic spine $(n=14,30.4 \%)$ and sacral $(n=11,23.9 \%)$. No patient had cervical involvement in our sample. The number of affected vertebrae ranged from 1 to 4 , and $8(17.4 \%)$ cases had multiple lesions (lesions $\geq 3$ ). Multiple myeloma was the most common neoplasm found in the analysis of vertebral biopsy, corresponding to 12 patients (26.1\%), followed by metastatic breast carcinoma, 9 (19.6\%), prostate cancer, 5 (10.9\%), and lung cancer, 5 (10.9\%). Seven patients (15.2\%) showed no neoplastic lesions.

Conclusions Epidemiological data was consistent with the literature data, except by histological type of the vertebral metastases, which multiple myeloma was the most prevalent in our study. This study is relevant because it is the first to assess vertebral metastases in the Brazilian North-Northeast.

\section{Introdução}

As metástases configuram o grupo mais comum dos tumores da coluna vertebral, com cerca de 18 a 25 mil novos casos diagnosticados por ano nos Estados Unidos. ${ }^{1,2}$ Sabe-se que até $10 \%$ dos pacientes com câncer desenvolvem lesões medulares secundárias sintomáticas, com vários níveis de envolvimento. ${ }^{3}$ Por isso, cirurgiões de coluna são frequentemente confrontados com decisões sobre a melhor forma de tratar os pacientes com estas lesões. Apesar de não representarem a cura, a ressecção e estabilização podem ter efeitos muito benéficos sobre o estado neurológico e funcional destes pacientes. ${ }^{4,5}$

A coluna vertebral é o sítio ósseo mais comumente acometido por metástases de neoplasias malignas. Sua incidência varia entre 30 e $90 \%$ em pacientes com câncer em estágio terminal. ${ }^{6-8} \mathrm{~A}$ disseminação das células neoplásicas para a coluna vertebral ocorre usualmente por via hematogênica, linfática, liquórica (rara) ou invasão direta por contiguidade. Destas, a via hematogênica é a principal e geralmente ocorre através do plexo venoso vertebral de Batson. ${ }^{7,9,10}$

Devido a sua menor morbimortalidade quando comparada à biópsia aberta, a biópsia percutânea tem-se tornado uma importante ferramenta de coleta de material do corpo vertebral e, consequentemente, uma notável ferramenta diagnóstica. A técnica foi descrita pela primeira vez em 1934, quando Ball et al. realizaram a primeira série de biópsia percutânea. ${ }^{11}$ Com uma série de publicações sobre o método, em 1948 Valls e Ottolen- ghi consagraram o seu uso na coluna vertebral, descrevendo a técnica como segura, confiável e com baixo índice de complicações. ${ }^{12}$

Com isso, o presente estudo objetiva analisar o perfil epidemiológico dos pacientes com suspeita de doença metastática na coluna vertebral, atendidos em hospital de referência em neurocirurgia da Bahia e submetidos à biópsia percutânea.

\section{Métodos}

Trata-se de um estudo observacional, descritivo e retrospectivo, realizado no hospital de referência neurocirúrgica do estado da Bahia (Hospital Geral Roberto Santos).

Os dados foram coletados em prontuários de pacientes submetidos à biópsia percutânea da coluna vertebral por suspeita de patologia metastática no período de março de 2013 a fevereiro de 2014. Não houve restrição de sexo ou idade.

Foram avaliadas variáveis tais como idade, sexo, neoplasia primária e data de diagnóstico, neoplasia secundária e data de diagnóstico, bem como níveis de acometimento em coluna vertebral. Das variáveis categóricas, foram obtidas as frequências, absolutas e percentuais. Das variáveis contínuas, foram obtidas as médias e o desvio padrão, em caso de distribuição normal, ou a mediana e os percentis, em caso de distribuição não normal.

Os dados obtidos foram analisados através do programa Statistical Package for the Social Sciences (SPSS) versão 17.0. 


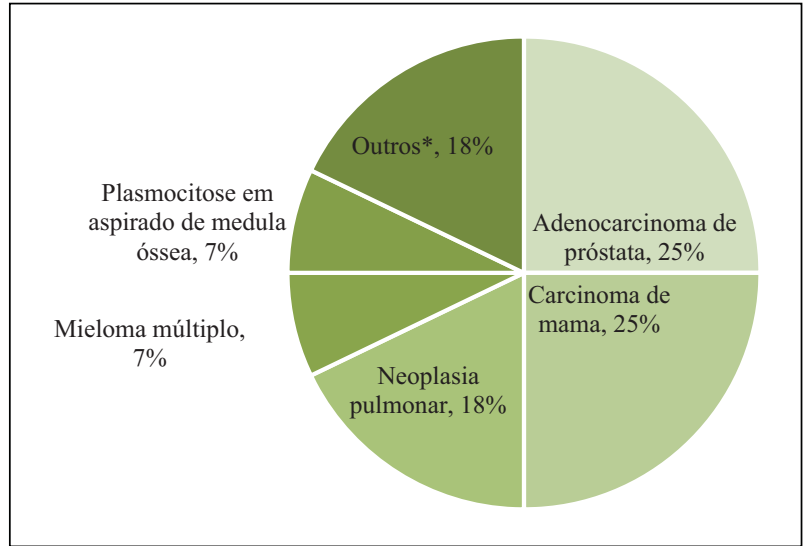

Fig. 1 Distribuição dos tipos de neoplasia nos 26 pacientes com diagnóstico anterior à biópsia vertebral. *Adenocarcinoma gástrico, carcinoma folicular da tireóide e neoplasia de endométrio: 1 caso.

\section{Resultados}

Foram incluídos 46 pacientes, dos quais 25 (54,3\%) eram do sexo masculino. A idade variou entre 16 e 81 anos, com média de 54,3 anos (desvio padrão: 15 anos) e mediana de 56 (intervalo interquartil - IIQ: 45-64 anos).

Mais da metade dos pacientes (56,2\%) possuíam diagnóstico de neoplasia anterior à biópsia vertebral, sendo os tipos mais comuns o adenocarcinoma de mama e próstata, seguidos pela neoplasia pulmonar ( - Fig. $\mathbf{1}$ ).

Em relação à topografia da lesão vertebral, a coluna lombar foi a mais frequente, estando acometida em 31 $(67,4 \%)$ casos, seguida pela torácica em $14(30,4 \%)$ e sacral em 11 (23,9\%). Nenhum paciente apresentou acometimento cervical em nossa amostra. 0 número de vértebras acometidas variou de 1 a 4 , com mediana de 2 (IIQ: 1 - 2), sendo que $8(17,4 \%)$ casos apresentavam lesões múltiplas ( $\geq 3$ lesões). $A$ fratura patológica, presente em 26 pacientes (56,5\%), foi mais comum que a fratura destrutiva, que ocorreu em 19 casos $(41,3 \%)$. No tocante aos níveis vertebrais, o nível mais alto foi T2 e o mais baixo, S2 (-Fig. 2).

O mieloma múltiplo foi a neoplasia mais comumente encontrada na análise do material de biópsia vertebral, correspondendo a 12 casos $(26,1 \%)$, seguido pela metástase de carcinoma de mama, 9 (19,6\%); de próstata, 5 (10,9\%); e pulmonar, 5 (10,9\%) (-Fig. 3 ).

A maior parte dos diagnósticos $(52,2 \%)$ foi dada apenas por meio do estudo histológico, sendo que $22(47,8 \%)$ necessitaram do estudo histológico e do imuno-histoquímico. Cabe ressaltar que em 7 pacientes (15,2\%) não foram identificadas alterações sugestivas de neoplasia em ambos os métodos.

O tempo entre o diagnóstico primário e o secundário variou de 3 dias a 4,6 anos, com mediana de 4 meses (IIQ: 35,5-431 dias).

\section{Discussão}

Embora a metástase na coluna vertebral possa ocorrer em qualquer faixa etária, a maior incidência é encontrada na de 40-65 anos, que corresponde ao período de maior risco do desenvolvimento de neoplasias malignas. ${ }^{13}$ Em nossa amostra, a faixa etária variou de 16 a 81 anos de idade, com mediana de 56 anos (IIQ: 45-64), concordante com dados da literatura. $^{14-22}$

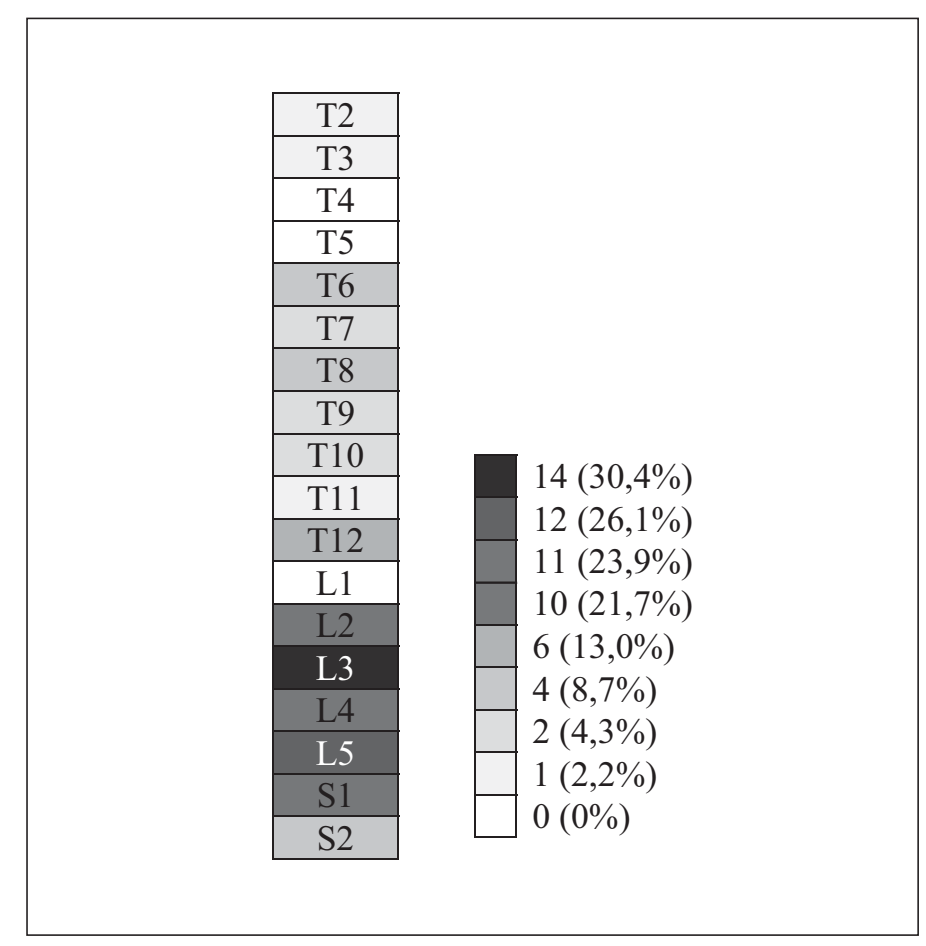

Fig. 2 Frequência do acometimento dos níveis vertebrais nos 46 pacientes com suspeita de lesão metastática. 


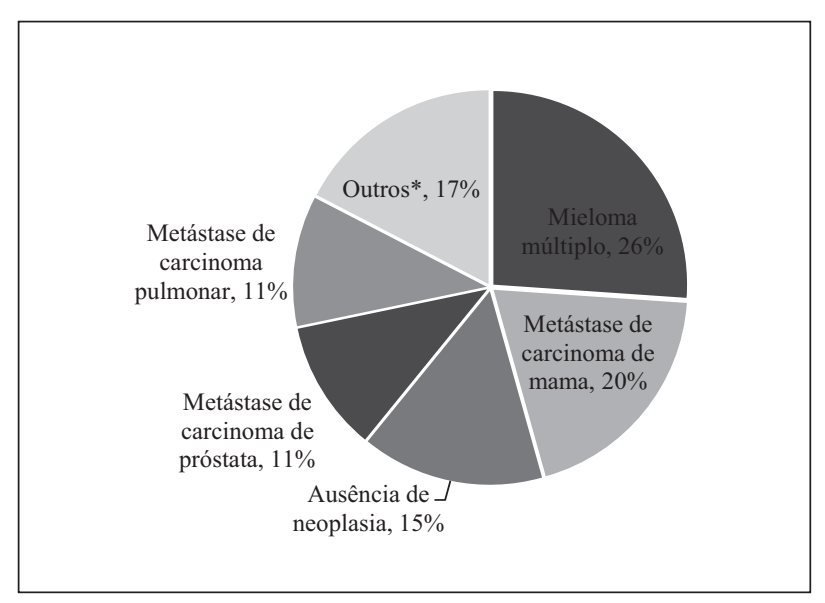

Fig. 3 Distribuição dos resultados da biópsia dos 46 pacientes com suspeita de metástase vertebral. *Plasmocitoma: 2 casos. Linfoma linfoplasmocítico, metástase de adenocarcinoma gástrico, metástase de carcinoma de tireoide, osteoblastoma, osteossarcoma periosteal e sarcoma de Ewing: 1 caso.

Em nosso estudo, observou-se uma discreta predominância do sexo masculino em relação ao sexo feminino (proporção de 1,2:1). Em revisão de literatura, encontramos que ambos os sexos são acometidos de maneira semelhante ${ }^{19,23,24}$, porém, em alguns estudos, há maior prevalência no sexo masculino, também de forma discreta. $^{7,14,15,25}$ Sciubba et al justificam que este fato provavelmente ocorre devido à prevalência ligeiramente maior de câncer de pulmão nos homens, e de câncer de próstata quando comparado ao de mama em mulheres. ${ }^{7}$

As metástases vertebrais ocorrem mais frequentemente na região lombar (70\%), seguida da torácica (20\%) e cervical $(10 \%){ }^{22}$ Sua distribuição está relacionada ao tamanho das vértebras, sendo que quanto maior o tamanho da vértebra, maior essa prevalência. Os dados encontrados no presente estudo corroboram aqueles citados na literatura, mostrando incidência de $67,4 \%$ na coluna lombar e nenhum acometimento na coluna cervical. Vale ressaltar entretanto, que as lesões mais sintomáticas são as localizadas na região torácica, representando aproximadamente $70 \%$ dos casos. Isso por que o canal vertebral é caracteristicamente mais estreito, havendo um menor espaço disponível para a medula espinhal nessa região. ${ }^{7,10,13,22}$ Observamos ainda que $17,4 \%$ dos casos apresentavam lesões metastáticas vertebrais múltiplas, número condizente com os dados do estudo de Byrne, que revelam incidência de $17-30 \%$ de lesões múltiplas demonstradas por ressonância magnética. ${ }^{26}$

As neoplasias de coluna mais comuns são as metástases do adenocarcinoma de mama, seguido pelas de neoplasias pulmonares, de próstata, dos rins e dos tumores hematopoiéticos, destacando-se o mieloma múltiplo e o linfoma. ${ }^{27}$ Essa distribuição reflete tanto a prevalência dessas neoplasias específicas, quanto a sua predileção para a disseminação óssea. Em nossa amostra, tivemos maior prevalência de metástases de mieloma múltiplo, seguida por adenocarcinoma de mama, dados que diferem da literatura, que apresenta o mieloma múltiplo de forma menos preva- lente. ${ }^{15,17,28,29}$ Entretanto, em recente estudo regional brasileiro, o mieloma múltiplo representou a segunda causa de doença metastática vertebral. ${ }^{22}$

\section{Conclusões}

O presente estudo encontrou dados epidemiológicos quanto a sexo, idade e local de lesão compatíveis com a literatura nacional e internacional. Contudo, o tipo histológico das neoplasias que cursaram com metástases vertebrais diferiu, sendo mieloma múltiplo o de maior prevalência em nosso estudo.

Apesar de termos algumas limitações, como a pequena amostra e o método retrospectivo, feito a partir da análise de prontuários, nosso estudo torna-se relevante por ser o primeiro a avaliar metástases vertebrais na região da coluna.

\section{Referências}

1 Schiff D. Spinal cord compression. Neurol Clin 2003;21(1):67-86, viii viii.

2 Gokaslan ZL, York JE, Walsh GL, et al. Transthoracic vertebrectomy for metastatic spinal tumors. J Neurosurg 1998;89(4):599-609

3 Grant R, Papadopoulos SM, Greenberg HS. Metastatic epidural spinal cord compression. Neurol Clin 1991;9(4):825-841

4 Colletti PM, Siegel HJ, Woo MY, Young HY, Terk MR. The impact on treatment planning of MRI of the spine in patients suspected of vertebral metastasis: an efficacy study. Comput Med Imaging Graph 1996;20(3):159-162

5 Fourney DR, Abi-Said D, Rhines LD, et al. Simultaneous anteriorposterior approach to the thoracic and lumbar spine for the radical resection of tumors followed by reconstruction and stabilization. J Neurosurg 2001;94(2, Suppl)232-244

6 Quraishi NA, Gokaslan ZL, Boriani S. The surgical management of metastatic epidural compression of the spinal cord. J Bone Joint Surg Br 2010;92(8):1054-1060

7 Sciubba DM, Petteys RJ, Dekutoski MB, et al. Diagnosis and management of metastatic spine disease. A review. J Neurosurg Spine 2010;13(1):94-108

8 Wong DA, Fornasier VL, MacNab I. Spinal metastases: the obvious, the occult, and the impostors. Spine 1990;15(1):1-4

9 Jacobs WB, Perrin RG. Evaluation and treatment of spinal metastases: an overview. Neurosurg Focus 2001;11(6):e10

10 Daniel JW, Veiga JCE. Diretrizes no tratamento das metástases epidurais da coluna vertebral: atualização. Arq Bras Neurocir 2007;26(3):93-110

11 Ball RP. Needle (aspiration) biopsy. J Am Med Assoc 1936;107(17): 1381

12 Valls J, Ottolenghi CE, Schajowicz F. Aspiration biopsy in diagnosis of lesions of vertebral bodies. J Am Med Assoc 1948;136(6): 376-382

13 Araujo JL, Veiga JC, Figueiredo EG, Barboza VR, Daniel JW, Panagopoulos AT. Management of metastatic spinal column neoplasms-an update. Rev Col Bras Cir 2013;40(6):508-514

14 Bernat JL, Greenberg ER, Barrett J. Suspected epidural compression of the spinal cord and cauda equina by metastatic carcinoma. Clinical diagnosis and survival. Cancer 1983;51(10):1953-1957

15 Chaichana KL, Pendleton C, Wolinsky J-P, Gokaslan ZL, Sciubba DM. Vertebral compression fractures in patients presenting with metastatic epidural spinal cord compression. Neurosurgery 2009; 65(2):267-274, discussion 274-275

16 Klekamp J, Samii H. Surgical results for spinal metastases. Acta Neurochir (Wien) 1998;140(9):957-967 
12 Perfil epidemiológico das metástases para coluna vertebral Pamplona et al.

17 Lu C, Gonzalez RG, Jolesz FA, Wen PY, Talcott JA. Suspected spinal cord compression in cancer patients: a multidisciplinary risk assessment. J Support Oncol 2005;3(4):305-312

18 Rose PS, Laufer I, Boland PJ, et al. Risk of fracture after single fraction image-guided intensity-modulated radiation therapy to spinal metastases. J Clin Oncol 2009;27(30):5075-5079

19 Shah AN, Pietrobon R, Richardson WJ, Myers BS. Patterns of tumor spread and risk of fracture and epidural impingement in metastatic vertebrae. J Spinal Disord Tech 2003;16(1):83-89

20 Talcott JA, Stomper PC, Drislane FW, et al. Assessing suspected spinal cord compression: a multidisciplinary outcomes analysis of 342 episodes. Support Care Cancer 1999;7(1):31-38

21 Taneichi H, Kaneda K, Takeda N, Abumi K, Satoh S. Risk factors and probability of vertebral body collapse in metastases of the thoracic and lumbar spine. Spine 1997;22(3):239-245

22 Filho ESV, Tardini R, Abreu LC, de Motter BV, Adami F, Rodrigues LMR. Estudo epidemiológico de 55 pacientes portadores de doença vertebral metastática sintomática em Santo André - SP, Brasil. Coluna/Columna 2013;12(1):32-35

23 Helweg-Larsen S, Hansen SW, Sørensen PS. Second occurrence of symptomatic metastatic spinal cord compression and findings of multiple spinal epidural metastases. Int J Radiat Oncol Biol Phys 1995;33(3):595-598

24 Helweg-Larsen S, Sørensen PS, Kreiner S. Prognostic factors in metastatic spinal cord compression: a prospective study using multivariate analysis of variables influencing survival and gait function in 153 patients. Int J Radiat Oncol Biol Phys 2000;46(5): 1163-1169

25 Husband DJ, Grant KA, Romaniuk CS. MRI in the diagnosis and treatment of suspected malignant spinal cord compression. $\mathrm{Br}$ J Radiol 2001;74(877):15-23

26 Byrne TN. Spinal cord compression from epidural metastases. N Engl J Med 1992;327(9):614-619

27 Joaquim AF, Alexandre F, Maturana DP, Zambelli HJL, Maldaun MVC. Metástases na coluna vertebral. Rev Neurocienc 2007:240-245

28 Levack P, Graham J, Collie D, et al; Scottish Cord Compression Study Group. Don't wait for a sensory level-listen to the symptoms: a prospective audit of the delays in diagnosis of malignant cord compression. Clin Oncol (R Coll Radiol) 2002;14(6):472-480

29 Roth SE, Mousavi P, Finkelstein J, Chow E, Kreder H, Whyne CM. Metastatic burst fracture risk prediction using biomechanically based equations. Clin Orthop Relat Res 2004;(419):83-90 